Cite this: Phys. Chem. Chem. Phys., 2011, 13, 11351-11358

www.rsc.org/pccp

PAPER

\title{
Excitonic effects in two-dimensional vibrational spectra of liquid formamide $\dagger$
}

\author{
Alexander Paarmann, ${ }^{a b}$ Manuela Lima, ${ }^{c}$ Riccardo Chelli, ${ }^{c d}$ Victor V. Volkov, ${ }^{c}$ \\ Roberto Righini ${ }^{c d}$ and R. J. Dwayne Miller*ae
}

Received 23rd December 2010, Accepted 19th April 2011

DOI: $10.1039 / \mathrm{c0cp02961k}$

The linear and two-dimensional infrared (2DIR) responses of the amide I vibrational mode in liquid formamide are investigated experimentally and theoretically using molecular dynamics simulations. The recent method based on the numerical integration of the Schrödinger equation is employed to calculate the 2DIR spectra. Special attention is devoted to the interplay of the structural dynamics and the excitonic nature of the amide I modes in determining the optical response of the studied system. In particular, combining experimental data, simulated spectra and analysis of the simulated atomic trajectory in terms of a transition dipole coupling model, we provide a convincing explanation of the peculiar features of the 2DIR spectra, which show a substantial increase of the antidiagonal bandwidth with increasing frequency. We point out that, at variance with liquid water, the 2DIR spectral profile of formamide is determined more by the excitonic nature of the vibrational states than by the fast structural dynamics responsible for the frequency fluctuations.

\section{Introduction}

Coherent multidimensional spectroscopies ${ }^{1-3}$ have emerged as a powerful tool for studying structural and dynamical aspects of the matter. Among these methodologies, two-dimensional infrared (2DIR) spectroscopy allows investigation of structural dynamics, intermolecular coupling, dephasing and relaxation mechanisms in a wide class of molecular systems. Important applications pertain to the study of polypeptides, ${ }^{4-19}$ proteins, ${ }^{20-25}$ DNA $^{26-28}$ and liquids. ${ }^{29-39}$ In polypeptides and proteins, structural changes are slow and the infrared (IR) lineshapes are often dominated by excitonic effects indicative of secondary

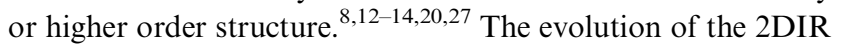
spectra can then be used to track conformational changes on time scales of tens and hundreds of picoseconds. ${ }^{4,8}$ For more site specific information, isotopic substitution is used ${ }^{25}$ to eliminate any resonant interaction. ${ }^{34-39}$ In this case, the

${ }^{a}$ Departments of Chemistry and Physics, University of Toronto, Toronto, ON M5S 3H6, Canada.

E-mail:dmiller@lphys.chem.utoronto.ca

${ }^{b}$ Fritz-Haber Institute of the Max-Planck Society, Faradayweg 4-6,

14195 Berlin, Germany

${ }^{c}$ European Laboratory for Non-Linear Spectroscopy (LENS),

via Nello Carrara 1, I-50019 Sesto Fiorentino, Italy

${ }^{d}$ Dipartimento di Chimica, Università di Firenze, via della Lastruccia 3, I-50019 Sesto Fiorentino, Italy

${ }^{e}$ Max Planck Group for Atomically Resolved Dynamics,

Department of Physics, University of Hamburg,

Centre for Free Electron Laser Science, DESY, Germany

$\dagger$ Electronic supplementary information (ESI) available. See DOI: $10.1039 / \mathrm{c} 0 \mathrm{cp} 02961 \mathrm{k}$
2DIR spectra are sensitive to the most immediate structural environment and the evolution of the 2DIR cross peaks can reveal the structural dynamics of labelled sites in close analogy with the two-dimensional NMR technique. In liquids, the 2DIR lineshapes and their evolution are dictated by the sub-picosecond dynamics of the local structure. In other words, the structural information gained from most 2DIR studies can be roughly separated into two major regimes: slow structural evolution on large length scales and fast structural dynamics on short length scales. For systems with extended H-bond networks, it would be interesting to also gain access to the dynamics on intermediate length scales, because the characteristics of the network become important in these regimes. Only most recently, it was shown that the 2DIR spectrum of liquid water can be sensitive to intermediate length scales ${ }^{30}$ through the delicate balance of local frequency fluctuations and intermolecular couplings. ${ }^{40}$ At room temperature, the spectra are still dominated by local structural dynamics, whereas at lower temperature delocalization of the vibrational excitations becomes important and the 2DIR spectra report on the dynamics of structural correlations between 15-20 molecules. ${ }^{30}$

In this respect, liquid formamide (FA) is an ideal model system. It can be considered as the simplest monomer unit that bridges liquid behavior to that of highly structured biomolecules. The amide I vibrational mode is relatively insensitive to the local environment (electric field fluctuations and $\mathrm{H}$-bonding) as shown by the rather narrow IR linewidth of the ${ }^{12} \mathrm{C} \mathrm{FA}$ impurity in ${ }^{13} \mathrm{C}$ FA solvent reported in ref. 33 (FWHM $\sim 20 \mathrm{~cm}^{-1}$ ). On the other hand, the resonant intermolecular coupling is 
comparable to that occurring in liquid water due to the large transition dipole moment. This results in largely delocalized excitons at ambient conditions, ${ }^{33,41}$ whose dynamics are regulated by the time evolution of the spatial correlations of the molecules. These correlations are ultimately modulated by H-bond networking. In fact, the emerging picture ${ }^{33,41-48}$ for liquid FA is that of an extended H-bond network, mostly consisting of highly branched $\mathrm{H}$-bonded chains, with a minor appearance of ring-shaped oligomers and cyclic dimers. ${ }^{44,45}$ For this reason it is considered a well-structured liquid ${ }^{33,47}$ and often used as a model system for other H-bonded liquids.

The importance of intermolecular vibrational coupling of the amide I mode in liquid FA was first discovered in polarized Raman experiments. ${ }^{42,49}$ In these measurements, the difference in peak position between IR, isotropic and anisotropic Raman signals, the so-called Raman noncoincidence effect, ${ }^{41,49-53}$ is indicative of the resonant vibrational interactions and formation of vibrational excitons. ${ }^{53}$ Even though some understanding about prominent structural domains can be extracted, all these studies are limited to time-averaged properties of the FA liquid structure.

Here, by using 2DIR spectroscopy and computational modeling, we investigate the interplay of the structural dynamics and the excitonic nature of amide I modes in the optical response of liquid FA. The analysis is based on previous work $^{33}$ where 2DIR pump-probe spectroscopy of neat FA and of a $1: 10{ }^{12} \mathrm{C}:{ }^{13} \mathrm{C}$ FA isotopic mixture, along with molecular dynamics (MD) simulations, were used to characterize the spectroscopic parameters of the amide I mode. The isotopic substitution allowed to distinguish between the local frequency fluctuations and the excitonic contributions to the linear and 2DIR lineshapes. The local structural dynamics were characterized in ref. 33 through appropriate modeling of the ${ }^{12} \mathrm{C}$ FA impurity spectra. We now extend the modeling to study and interpret the dynamics of the vibrational excitons in neat FA using the simulation procedure based on numerical integration of the Schrödinger equation (NISE) developed for the study of liquid water. ${ }^{31,32}$ In particular, combining experimental data, simulated spectra obtained from NISE approach and analysis of the MD atomic trajectories, we correlate the delocalized character of the vibrational excitations to the peculiar profile of the 2DIR spectra.

The article is organized as follows. In section 2, we describe the procedures adopted in the experiments and in theoretical modeling. In section 3 , we report the basic results and compare the experimental outcomes to the calculations. A thorough discussion of the data, supported by further calculations in terms of excitonic features of the system, is given in section 4 . Concluding remarks can be found in section 5 .

\section{Materials and methods}

\subsection{Experimental setup}

In the following we will report on 2DIR spectra of neat FA measured at 25 and $2{ }^{\circ} \mathrm{C}$ in the frequency range of the amide I mode. Measurements have been done at zero pump-probe delay time for parallel (XXXX) and perpendicular (XXYY) polarizations of pump and probe pulses. The XXXX polarized spectrum at room temperature has been published elsewhere. ${ }^{33}$ The 2DIR spectra were recorded in a spectral hole-burning pump-probe setup. A Ti:Sapphire amplified laser system pumps two optical parametric amplifiers (OPAs) followed by difference frequency generation (DFG) in a $\mathrm{Ag}_{2} \mathrm{GaS}$ crystal to generate broadband pump and probe pulses at $\lambda=6 \mu \mathrm{m}$ with a bandwidth of $\sim 200 \mathrm{~cm}^{-1}$. The pump pulse passes a Fabry-Perot filter reducing the pump bandwidth to $15 \mathrm{~cm}^{-1}$. In the hole burning experiment, the center wavelength of the pump pulse is scanned by adjusting the separation between the etalon mirrors. A wave plate in the pump beam allows switching between all parallel and perpendicular polarization of pump and probe pulses. The scanning-pump broadband-probe signal as a function of pump-probe delay time and pump wavelength is recorded spectrally dispersed using an imaging monochromator and a MCT detector array. The 2DIR spectra are generated by plotting the pump-probe signal as a function of the pump (etalon) and probe (spectrometer) frequencies. The time and spectral resolutions of these hole burning experiments are inherently limited through the use of an etalon. In the given experiments, the spectral resolution is $15 \mathrm{~cm}^{-1}$ and the temporal resolution is $\sim 0.7$ ps. More details on the experimental procedure can be found in ref. 33 .

\subsection{Computational techniques and modeling}

The nonlinear vibrational response of the amide I mode in FA is calculated using the NISE method presented in ref. 32 . The overall steps of the algorithm are identical to those employed to calculate the 2DIR response of the $\mathrm{OH}$ stretching vibration in liquid water. ${ }^{32}$ First we determine the parameters entering in the vibrational Hamiltonian (electrostatic map, anharmonicity, etc.), then we calculate the time-dependent vibrational Hamiltonian using the MD simulation trajectory and, finally, we calculate the optical response from the vibrational Hamiltonian. While the final step is generic and identical for different systems, the first two steps are specific to the molecular system under study, to the specific vibrational mode and to the model employed for exciton coupling ${ }^{54}$ as well. The system dynamics provided by the MD simulation allow us to employ an effective time-dependent vibrational Hamiltonian in the local molecular basis ${ }^{32}$ (in our case the amide I mode of FA):

$$
\begin{aligned}
\hat{H}(t)= & \sum_{m=1}^{N} \omega_{m}(t) \hat{B}_{m}^{\dagger} \hat{B}_{m}+\sum_{m=1}^{N} \sum_{n \neq m}^{N} J_{m n}(t) \hat{B}_{m}^{\dagger} \hat{B}_{n} \\
& -\frac{\Delta}{2} \sum_{m=1}^{N} \hat{B}_{m}^{\dagger} \hat{B}_{m}^{\dagger} \hat{B}_{m} \hat{B}_{m},
\end{aligned}
$$

where $\omega_{m}(t)$ is the fundamental transition frequency of the $m$ th amide I mode at time $t, \hat{B}_{m}^{\dagger}$ and $\hat{B}_{m}$ are the creation and annihilation operators of the mode, respectively, $J_{m n}(t)$ is the resonant vibrational coupling between amide I modes $m$ and $n$, and $\Delta$ is a constant anharmonicity shift. We note that the diagonal character of the anharmonic term allows for exact solution of the anharmonic propagation ${ }^{55}$ in the split-operator procedure. ${ }^{32}$ Clearly, the sums in eqn (1) run over the $N$ amide I modes of the simulated sample. Typically, the vibrational Hamiltonian parameters such as the transition dipole moments [entering in the estimate of $J_{m n}(t)$ ], $\omega_{m}(t)$ and $\Delta$ are acquired 
from quantum mechanical calculations. ${ }^{32,56-59}$ Here, we follow a much simpler approach. The estimate of $\omega_{m}(t)$ is based on an empirical electrostatic map, ${ }^{33,60}$ assuming a linear dependence of the Stark frequency shift ${ }^{61}$ by the component of the electric field along the direction of the transition dipole moment, ${ }^{33}$ i.e.,

$$
\omega_{m}(t)=\omega_{0}-k \mathbf{E}_{m}(t) \cdot \mathbf{e}_{m}(t)
$$

where $\omega_{0}$ is a constant, $k$ is the Stark coefficient, and $\mathbf{E}_{m}(t)$ is the electric field acting on the transition dipole moment of the $m$ th amide I mode ${ }^{62}$ whose direction and orientation is represented by the unit vector $\mathbf{e}_{m}(t)$. Following Torii and Tasumi, ${ }^{63}$ the transition dipole moment is assumed to be fixed with respect to the molecular frame. Thus, the full map depends only on two independent parameters to be determined empirically: the Stark coefficient $k$ and the reference frequency $\omega_{0}$. In order to acquire these parameters, the linear IR response of the ${ }^{12} \mathrm{C}$ amide I mode measured for a $1: 10$ ${ }^{12} \mathrm{C}:{ }^{13} \mathrm{C}$ FA isotopic mixture (from ref. 33) is fitted using the NISE formalism. ${ }^{32}$ The ${ }^{12} \mathrm{C}$-labeled molecules, because of their low concentration in the isotopic mixture, can be considered isolated species in a solvent made of ${ }^{13} \mathrm{C}$-labeled molecules. For this reason, the amide I mode of these molecules will be excitonically uncoupled from the vibrational modes of the solvent, thus allowing a fit to $k$ and $\omega_{0}$ by neglecting the solute-solvent resonant coupling $\left[J_{m n}(t)=0\right.$ in eqn (1)]. These linear response calculations explicitly treat the non-adiabatic dynamics of the vibrational excitonic system. The effect of the lifetime of the first excited vibrational state was also considered introducing a homogeneous broadening corresponding to a lifetime $T_{1}$ of $1.4 \mathrm{ps}$, as recovered from broadband pumpprobe data. ${ }^{33}$ The value of $\omega_{0}$ is $1738 \mathrm{~cm}^{-1}$, while the value of $k$ is very close to that found from a fit ${ }^{64}$ of the measured 2DIR spectra of the FA isotopic mixture ${ }^{33}\left(k=3168 \mathrm{~cm}^{-1} \mathrm{e} \mathrm{a}_{0} \mathrm{E}_{\mathrm{h}}^{-1}\right)$.

The calculated linear IR spectrum of the ${ }^{12} \mathrm{C}$ amide I mode in the FA isotopic mixture ${ }^{65}$ is shown in Fig. 1 along with the experimental counterpart. We notice that the fitted Stark coefficient directly leads to an amplitude of the frequency fluctuations of about ${ }^{33} 12.4 \mathrm{~cm}^{-1}$ (FWHM of the frequency distribution equal to $24.8 \mathrm{~cm}^{-1}$ ). With a FWHM of $18 \mathrm{~cm}^{-1}$

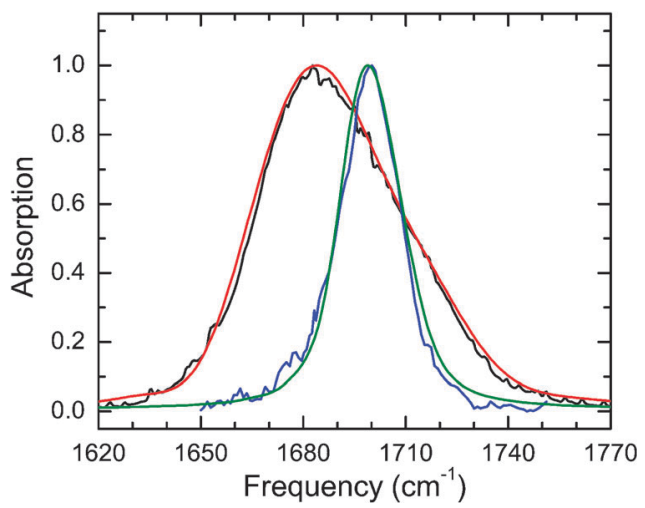

Fig. 1 Linear IR spectra of the ${ }^{12} \mathrm{C}$ amide I mode in neat FA and in $1: 10{ }^{12} \mathrm{C}:{ }^{13} \mathrm{C}$ FA isotopic mixture. Black line: experimental spectrum in neat FA; blue line: experimental spectrum in FA isotopic mixture; red line: calculated spectrum in neat FA; green line: calculated spectrum in FA isotopic mixture. The experimental data are taken from ref. 33. for the linear IR spectrum, the effect of motional narrowing is evident. However, approximately $20 \%$ of the motional narrowing is compensated by homogeneous broadening due to the finite lifetime of the transition $\left(T_{1}=1.4 \mathrm{ps}\right)$.

The next parameter to be determined in the vibrational Hamiltonian is the coupling factor $J_{m n}(t)$. This parameter is configuration dependent and, according to previous modeling, ${ }^{33,60}$ is calculated using the transition dipole coupling model. ${ }^{32,66-68}$ In spite of the underlying approximations, the transition dipole coupling model has been revealed to be a reliable approach for reproducing the basic spectral features (including the Raman noncoincidence effect) of strongly excitonic systems, such as liquid $\mathrm{FA},{ }^{41}$ methanol and acetone. ${ }^{53}$ In the transition dipole coupling model, the exciton coupling between two normal modes occurs through the electric interaction of their transition dipole moments, which depends on their mutual arrangement and on their amplitude $\mu$. In the present model, amplitude fluctuations and anharmonicity of the transition dipole moment are neglected. On the basis of this assumption, the time dependence of the transition dipole moment arises only from the orientational dynamics of the FA molecules, which was shown to reproduce correctly the spectral properties of liquid FA. ${ }^{41}$ The amplitude $\mu$ is determined by fitting the experimental linear IR spectrum of neat FA with that calculated using NISE approach [including intermolecular couplings, i.e. $\left.J_{m n}(t) \neq 0\right]$. In this calculation, we have used the value of $k$ obtained by the procedure described above. Good agreement between experiment and calculation is found for $\mu=3.46 \mathrm{D}^{-1} \mathrm{amu}^{-1 / 2}$ which is slightly larger than the value of $3.3 \mathrm{D}^{-1} \mathrm{amu}^{-1 / 2}$ extracted from a static exciton basis analysis. ${ }^{33}$ Both values are in good agreement with $a b$ initio calculations. ${ }^{15}$ According to results of a fit of 2DIR spectra of the FA isotopic mixture, ${ }^{33}$ the vibrational anharmonicity ( $\Delta$ in eqn (1)) has been assumed to be $18 \mathrm{~cm}^{-1}$. Careful inspection of the 2DIR spectrum of the FA isotopic mixture $^{33}$ also shows no detectable change of this value across the spectrum, well justifying our assumption of a constant value for $\Delta$.

The experimental and calculated linear IR spectra of neat FA are reported in Fig. 1. As for the isotopic mixture case, motional narrowing and $T_{1}$ lifetime affect the lineshape significantly. However, it is noteworthy that in this case motional narrowing contributes to the bandwidth by only $10 \%$, i.e., significantly less than in the IR spectrum of the isotopic mixture. This is due to the large line broadening arising from excitonic coupling occurring in neat FA. The pronounced asymmetry of the lineshape with a tail into the high frequency region is natively reproduced in our calculations. The microscopic origin of the asymmetry is not entirely clear, but it is certainly related to the coherence size and IR activity of the excitonic modes. ${ }^{41}$

The MD simulation has been performed with the ORAC program $^{69}$ on a sample of 98 FA molecules using the same potential model and procedures described in ref. 33. In short, after equilibrating the system at the temperature of $25{ }^{\circ} \mathrm{C}$ in the microcanonical ensemble (fixing the density at the value of a previous simulation ${ }^{33}$ ), a production run of $50 \mathrm{ps}$ has been carried out recording the atomic Cartesian coordinates every 2 fs. In section 4 we will also report data obtained from an 
earlier MD simulation of a sample of 320 molecules. ${ }^{33}$ This difference will be noted below to alert the reader.

The vibrational Hamiltonian of eqn (1) has been constructed in steps of $4 \mathrm{fs}$ for the whole MD trajectory. A total of 900 nonlinear signal trajectories (300 and 600 for parallel and perpendicular polarizations, respectively) for coherence times $t_{1}$ and $t_{3}^{32}$ up to 1 ps per population time $t_{2}$ have been calculated and averaged appropriately.

\section{Results}

The experimental and calculated 2DIR spectra of neat FA recorded with XXXX polarization and zero pump-probe delay time are shown in Fig. 2. As expected, the spectra exhibit two peaks, the fundamental $0 \rightarrow 1$ transition on the diagonal and the red shifted $1 \rightarrow 2$ excited state absorption peak. Both peaks are tilted and stretched along the diagonal, even though to a different degree, indicating initial inhomogeneity of the vibrational excitations. This feature is less pronounced in the experimental spectrum, pointing to a possible excess of inhomogeneity in the simulated sample. However, we must consider that no corrections are introduced in the calculated spectrum to account for the limited spectral and temporal resolution of the 2DIR pump-probe experiments. In fact, the reduced initial inhomogeneity observed in the experiment is, in part, caused by the $0.7 \mathrm{ps}$ time resolution resulting in temporal integration over parts of the spectral diffusion dynamics. Such an effect is expected to be significant because the dynamics of FA are comparable to this time resolution, as revealed by the correlation time (of about $0.9 \mathrm{ps}$ ) extracted from the frequency fluctuation correlation function of liquid FA. ${ }^{33}$ Consistently, the spectral diffusion of the simulated 2DIR spectra (see supplementary material) indicates an almost complete loss of inhomogeneity before $1 \mathrm{ps}$. Specifically, the overall spectral diffusion time scale is $200-400 \mathrm{fs}$, whereas a somewhat faster loss of correlation is observed on the blue side of the spectrum (200 fs) compared to the red side of the spectrum (300-400 fs). These spectral differences are mostly washed out by 600 fs when most frequency correlation has decayed. The basic features of the experimental spectrum are reproduced satisfactorily in the calculation, in particular the different tilt of the $0 \rightarrow 1$ and $1 \rightarrow 2$ transition peaks and

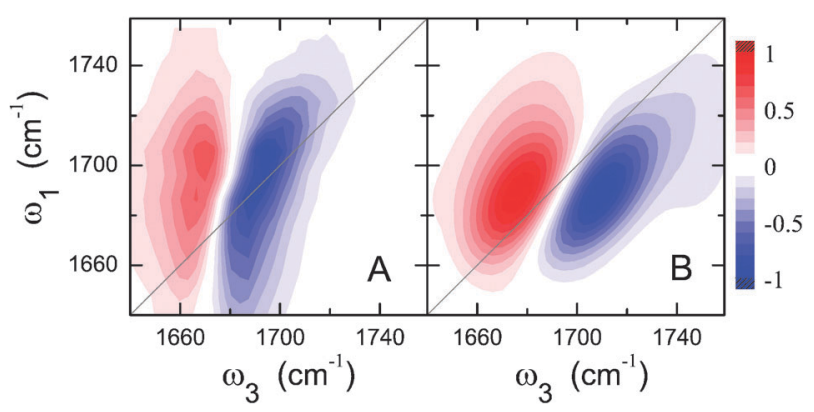

Fig. 2 Panel A: experimental 2DIR spectrum of neat FA recorded in XXXX polarization condition. Panel $B$ : 2DIR spectrum calculated at the same polarization condition. $\omega_{1}$ and $\omega_{3}$ refer to the excitation (pump) and detection (probe) frequencies, respectively. The experimental spectrum is taken from ref. 33. the larger antidiagonal width on the blue side of the $0 \rightarrow 1$ transition peak. This latter feature is consistently observed in these experiments. ${ }^{33}$

Insightful information on the origin of the 2DIR band profiles and their evolution can be gained by the comparison of the 2DIR spectra at different polarization conditions. The 2DIR spectra calculated with XXXX and XXYY polarization conditions are shown in Fig. 3. A pronounced noncoincidence of the lineshapes is observed. The XXYY polarized spectrum exhibits an overall reduced inhomogeneity with respect to the XXXX polarized one. Moreover the antidiagonal width on the blue side of the spectrum appears significantly enhanced in the XXYY polarization. This 2DIR noncoincidence effect is likely related to the Raman noncoincidence effect observed in liquid FA. ${ }^{41,49}$ The same trends for the change in 2DIR lineshape are also observed in the experiment. In Fig. 4, we show the 2DIR spectra measured in both XXXX and XXYY polarization conditions for temperatures of 25 and $2{ }^{\circ} \mathrm{C}$. At room temperature the difference between the two polarization conditions is rather subtle. This is likely caused by the combination of fast spectral diffusion and limited time resolution of the experiment. Since most initial inhomogeneity is lost in the XXXX polarized spectrum, the further reduction of the inhomogeneity in the XXYY polarized spectrum is necessarily not very pronounced though still detectable. However, in the 2DIR spectra at lower temperature the effect is very clear. At $2{ }^{\circ} \mathrm{C}$, the initial inhomogeneity in the XXXX polarized spectrum is stronger and, therefore, the difference with the XXYY polarized spectrum is more evident. These data will be employed below (section 4) to discuss the origin of the 2DIR band profile.

\section{Discussion}

It is known that the linear IR lineshape of the amide I mode of neat FA is strictly related to the excitonic nature of the vibrational $\operatorname{states}^{33,41}$ (see also Fig. 1). However, correlating the structural features of liquid FA to its IR spectrum is not an easy task, especially because the linewidth is broadened by disorder effects of both static and dynamic origin that produce large spectral overlaps between the different components. In this respect, the 2DIR spectroscopy may provide useful insights since it can disentangle the various spectral contributions. In principle, the antidiagonal width of the 2DIR fundamental peaks at zero pump-probe delay time and the evolution

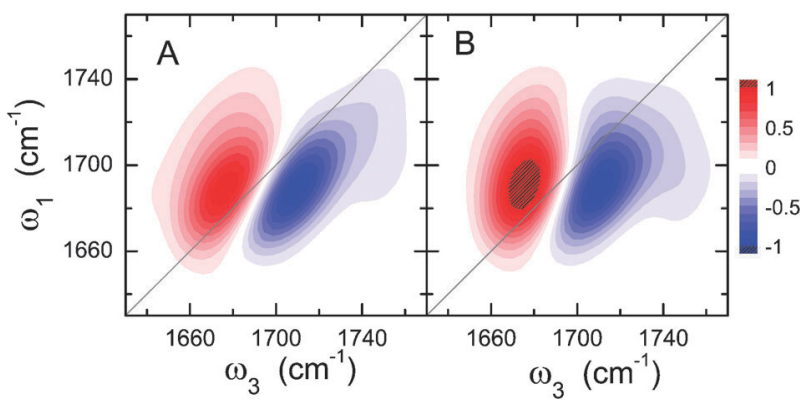

Fig. 3 2DIR spectra of neat FA calculated in XXXX and XXYY polarization conditions (panels $A$ and $B$, respectively). $\omega_{1}$ and $\omega_{3}$ refer to the excitation and detection frequencies, respectively. 


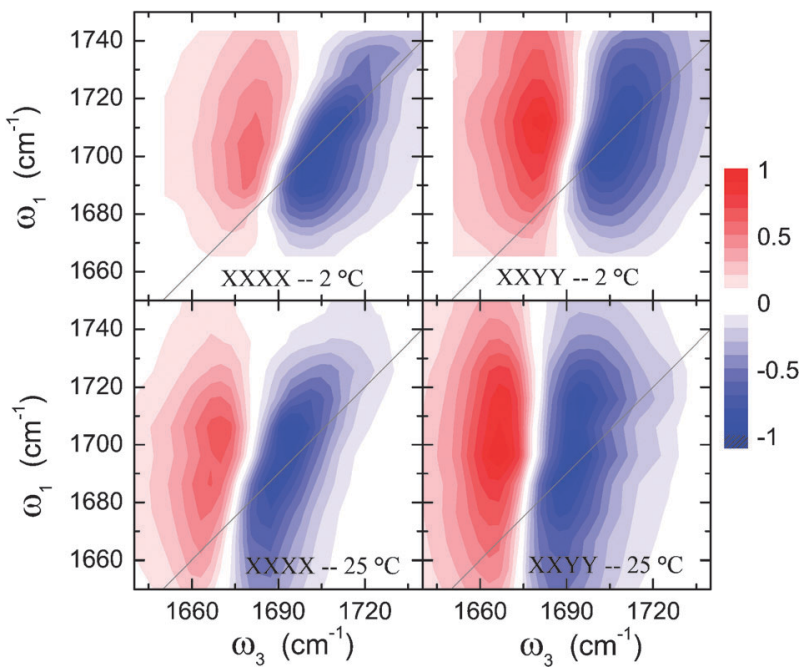

Fig. 4 2DIR spectra of neat FA measured in XXXX and XXYY polarization conditions for different temperatures (see panels). $\omega_{1}$ and $\omega_{3}$ refer to the excitation and detection frequencies, respectively.

of the lineshapes with pump-probe delay time (see supplementary material) can have two origins in excitonic systems: the rapid fluctuations of the transition frequencies and the energy transfer between different excitonic modes. The former case is related to the standard spectral diffusion picture. ${ }^{54}$ The fast components of the frequency fluctuation correlation function broaden the antidiagonal linewidth at the initial time (motional narrowing limit), whereas the slower components can be observed as the evolution of the 2DIR lineshape with pump-probe delay time. The second case (energy transfer among excitons) is instead related to coupling strength which mostly depends on the arrangement of the transition dipole moments. The 2DIR lineshape and its evolution is caused by the emergence of coupling-induced off-diagonal cross peaks between the different vibrational modes. This effect is well-known for spectrally separated modes. ${ }^{70}$ The initial magnitude of the cross peaks is related to the spatial overlap of the excitonic wave functions (common ground state) and the time evolution reports on the vibrational coupling strength. The larger the coupling the faster the cross peaks grow in.

In the case of liquid FA, individual cross peaks cannot be resolved due to the continuous spectral distribution of interacting states. Statistically, distributed cross peaks would rather lead to an antidiagonal broadening of the lineshape, much like the shape produced by fast spectral diffusion. In principle, the initial cross peak structure as well as the evolution of the cross peaks can produce 2DIR lineshapes very similar to the situation of spectral diffusion of uncoupled chromophores.

On the basis of the previous discussion, two possible hypothesis can be formulated to explain the important spectral feature related to the increased antidiagonal linewidth on the blue side of the 2DIR spectrum of neat FA: the excitonic modes on the blue side of the spectrum (i) fluctuate in frequency more rapidly or (ii) show increased coupling-induced crosspeak intensity, compared to the modes of the red side of the spectrum. Case (i) reports on structural dynamics leading to a picture of more rapidly fluctuating structures. This interpretation would be strengthened if one could admit that the high frequency excitons involve prevalently molecules characterized by weaker intermolecular interactions, that allow for the formation of short living structural motifs. Case (ii), on the other hand, would be supported by the increased excitonic delocalization and the consequent enhanced overlap of the excitons in the blue side of the spectrum.

The 2DIR spectra for different polarization conditions, reported in Fig. 3 and 4, provide a support to the arguments of case (ii). It is well-known that the off-diagonal cross-peak amplitudes can be amplified using the XXYY polarization condition, because such condition reduces the intensities of the diagonal peaks. ${ }^{71}$ In fact, this effect is often used to enhance cross-peak features. ${ }^{18,26,27}$ To highlight the 2DIR lineshape differences between the two polarization conditions, we have analyzed the difference between the XXYY and XXXX polarized spectra. Such a difference spectrum (not shown) is too convoluted to be interpreted directly. Instead, we aim to evaluate if there is a spectral dependence in these differences, i.e., if they are stronger or weaker for certain parts of the spectrum. This aspect is well captured by integrating the 2DIR difference spectrum ${ }^{72}$ along the detection axis $\omega_{3}$ and plotting the integral value as a function of the excitation frequency $\omega_{1}$. To prevent cancellation between the positive and negative signal parts, the squared values of the difference spectrum are integrated. The resulting traces for the calculated and experimental data at room temperature are shown in Fig. 5. To compare the integrated differences to the spectral profiles, the equivalent quantity $\left(\omega_{3}\right.$ integration over the squared value of the 2DIR spectrum) for the XXXX polarized spectrum is also shown. From Fig. 5 it is clear that the most important differences between the 2DIR spectra in the two polarization conditions occur on the blue side of the spectrum, thus highlighting the existence of a direct spectral correlation between the initial antidiagonal linewidth and the XXXX-XXYY 2DIR difference spectrum. Such a correlation suggests that the 2DIR lineshapes are dominated by coupling induced cross peaks. In this case, the dynamics of the 2DIR lineshape would only be related to the excitonic interactions (cross-peak growing) rather than to the structural dynamics in the liquid. This interpretation is further supported by the

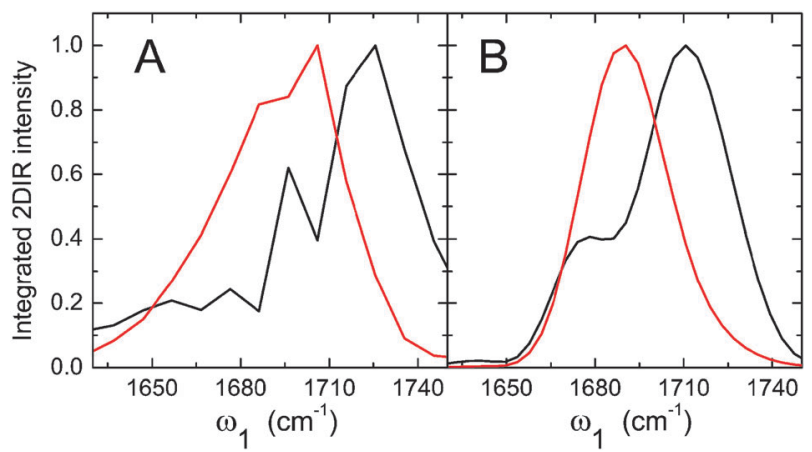

Fig. 5 Integrated 2DIR difference spectra of neat FA (black lines). The squared values of the 2DIR XXXX-XXYY difference spectra are integrated along $\omega_{3}$ (detection frequency) and plotted as a function of $\omega_{1}$ (excitation frequency). The equivalent integration for the XXXX 2DIR spectra is also shown (red lines). Panel $A$ : experiment at room temperature. Panel B: simulation. 
time scales of 2DIR lineshape evolution (see supplementary material). The energy transfer time, measured by pumpprobe polarization anisotropy (not shown), is about $200 \mathrm{fs}$ ( $300 \mathrm{fs}$ in the case of the simulated sample), which is in good agreement with the overall dynamics of the 2DIR lineshape evolution, thus suggesting that indeed the 2DIR lineshapes are dominated by vibrational coupling effects. Even though the similarity of the time scales is intriguing, such direct correlation between spectral diffusion and energy transfer is not necessarily justified. In fact, in liquid water several different regimes of spectral diffusion-energy transfer time ratios have been observed. ${ }^{30}$ In particular, at low temperatures 2DIR correlations decay slower than energy transfer processes.

Furthermore, in the scenario (ii), a larger contribution of cross peaks on the blue side of the 2DIR spectra would point to a stronger coupling between those excitonic states. We notice that two excitons are strongly coupled when the number of common local oscillators is large or, in other words, when their spatial overlap is relevant. Therefore, a stronger coupling of the excitonic modes in the blue frequency region should be correlated to a larger delocalization of those modes with respect to the modes in the red spectral range. This would in turn be reflected on the exciton size as probed by the frequency-dependent participation number, ${ }^{40}$ which is defined as

$$
\eta(\omega)=\frac{\left\langle\sum_{m=1}^{N}\left(\sum_{i=1}^{N} \Phi_{i m}^{4}\right)^{-1} \delta\left(\omega-\omega_{m}\right)\right\rangle}{\left\langle\sum_{n=1}^{N} \delta\left(\omega-\omega_{n}\right)\right\rangle},
$$

where $\omega_{m}$ is the frequency of the $m$ th exciton, $\Phi_{i m}$ is the eigenvector representing the contribution of the $i$ th local oscillator to the $m$ th exciton and the angular brackets indicate an average over the configurations. Eigenvectors and eigenvalues (frequencies) are obtained by diagonalizing the vibrational Hamiltonian matrix calculated from the simulated trajectory. The average value of the participation number ${ }^{73}$ is 65 , about $8 \%$ greater than that obtained in ref. 33 . This is due to the slightly larger transition dipole moment obtained in the present study (3.46 vs. $3.3 \mathrm{D}^{-1} \mathrm{amu}^{-1 / 2}$ ). In Fig. 6 we report $\eta(\omega)$. More precisely, in order to better compare the differences between blue and red frequency regions, we report the functions $\eta_{\text {blue }}(\omega)=\eta\left(\omega+\omega_{\max }\right)$ and $\eta_{\text {red }}(\omega)=\eta\left(\omega_{\max }-\omega\right)$, both defined for $\omega \geq 0$, where $\omega_{\max }$ corresponds to the frequency of the maximum IR intensity of neat $\mathrm{FA}^{74}\left(\omega_{\max }=1684 \mathrm{~cm}^{-1}\right)$. The large delocalization observed for blue excitons is clearly consistent with the above case (ii).

It is however important to remark that the excitonic delocalization revealed by the frequency-dependent participation number, albeit necessary, is not sufficient alone for observing excitonic coupling, that, on the other side, is involved more directly in the 2DIR signal. From the computational point of view we can probe excitonic coupling (necessary for observing a cross-peak signal) by using suitable functions aimed at quantifying spatial overlap between pairs of excitons. A quite

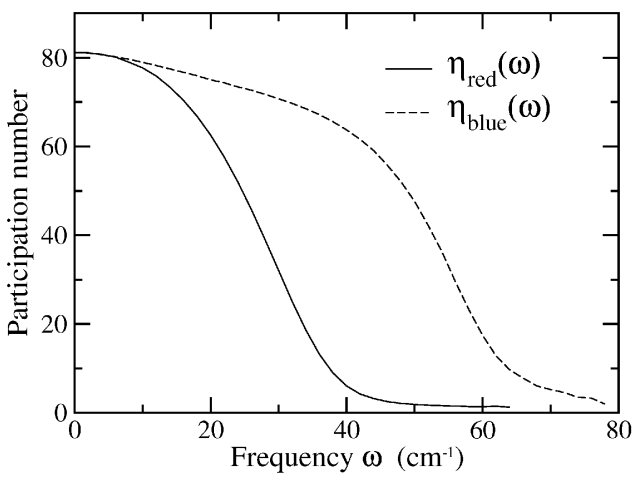

Fig. 6 Frequency-dependent participation number, $\eta(\omega)$, of neat FA calculated from eqn (3). For reasons explained in the text, we have drawn the functions $\eta_{\text {blue }}(\omega)=\eta\left(\omega+\omega_{\text {max }}\right)$ and $\eta_{\text {red }}(\omega)=\eta\left(\omega_{\max }-\omega\right)$, with $\omega_{\max }=1684 \mathrm{~cm}^{-1}$.

natural quantity is the dot product between exciton eigenvectors expressed as a function of their frequencies: ${ }^{73}$

$$
s\left(\omega_{a}, \omega_{b}\right)=\frac{\left\langle\sum_{m=1}^{N} \sum_{n \neq m}^{N}\left|\sum_{i=1}^{N} \Phi_{i m} \Phi_{i n}\right| \delta\left(\omega_{a}-\omega_{m}\right) \delta\left(\omega_{b}-\omega_{n}\right)\right\rangle}{\left\langle\sum_{m=1}^{N} \sum_{n \neq m}^{N} \delta\left(\omega_{a}-\omega_{m}\right) \delta\left(\omega_{b}-\omega_{n}\right)\right\rangle},
$$

where the notation introduced for eqn (3) has been used. In eqn (4), the absolute value is taken to ensure that $s\left(\omega_{a}, \omega_{b}\right)$ is independent on the phase of the excitons. Since $s\left(\omega_{a}, \omega_{b}\right)$ is defined in the context of a static picture of excitonic coupling, it quantifies directly the coupling contributing to the 2DIR spectrum at zero pump-probe delay time, with only an indirect effect on the spectra at later times. The plot of $s\left(\omega_{a}, \omega_{b}\right)$ is shown in Fig. 7. The black lines in the figure mark the frequency corresponding to the maximum intensity of the linear IR spectrum, $\omega_{\max }$, and hence separate the red frequency region

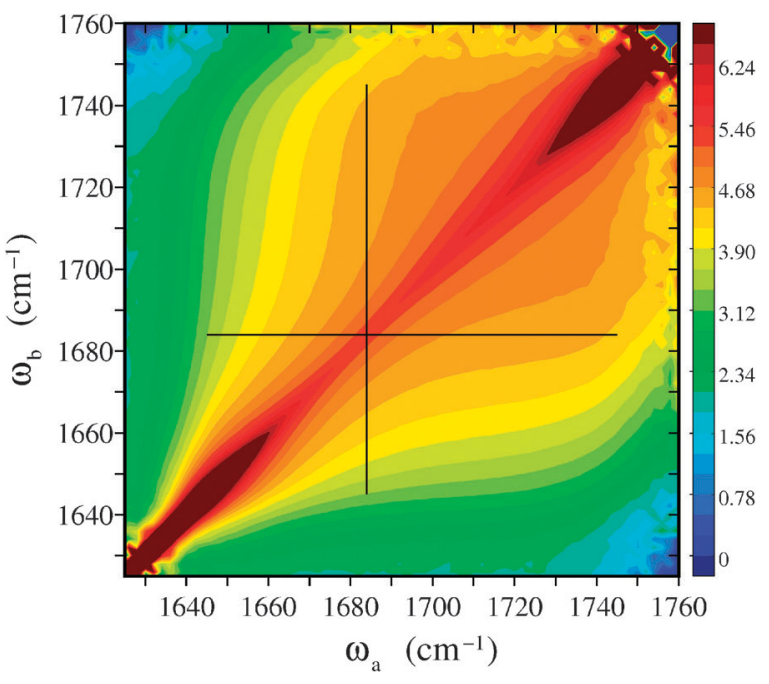

Fig. 7 Pair exciton coupling function, $s\left(\omega_{a}, \omega_{b}\right)$, as a function of the exciton frequencies for neat FA. The black lines correspond to the maximum intensity of the linear IR spectrum $\left(\omega_{a}=\omega_{\max }\right.$ and $\omega_{b}=\omega_{\max }$, where $\omega_{\max }=1684 \mathrm{~cm}^{-1}$ ). The chromatic scale reported on the right is arbitrary. 
from the blue one. By construction, $s\left(\omega_{a}, \omega_{b}\right)$ is symmetric with respect to frequency exchange. An important feature of $s\left(\omega_{a}, \omega_{b}\right)$ is the asymmetry in the distribution of the antidiagonal width across the two-dimensional plot, as highlighted by the two black lines in Fig. 7. It is evident that the coupling of the exciton states on the blue side covers a frequency range significantly greater than that involving the red side excitons. These outcomes are insightful for the interpretation of spectral features and, more strongly than the frequency-dependent participation number, support the interpretation of case (ii).

In order to assess the contribution of the structural dynamics to the 2DIR response, i.e. case (i) above, a simple test has been performed. We have artificially sped up and slowed down the structural dynamics by changing the time scale of the MD simulated trajectory used to construct the vibrational Hamiltonian. The same simulation trajectory has been used, but instead of extracting snapshots every $4 \mathrm{fs}$, they have been extracted every $2 \mathrm{fs}$ (slow dynamics) and every 8 fs (fast dynamics). In either case, the propagation step in the nonlinear signal calculation is taken to be $4 \mathrm{fs}$. In such a way we only manipulate the structural dynamics, leaving all other parameters unchanged. The resulting 2DIR spectra, calculated for XXXX and XXYY polarization conditions, are shown in Fig. 8. Overall, the differences between the spectra calculated at different dynamical regimes are not very large. This strengthens the previous arguments that the 2DIR spectra are mostly sensitive to the time-averaged structure rather than to the dynamics. However, few small systematic changes are observable. The overall spectral linewidth gets narrower on going from slow to fast dynamical systems because of the increased motional narrowing contribution. Note, however, that this effect is not very strong, thus supporting the view that the 2DIR lineshape is dominated by excitonic coupling effects. Secondly, the special feature on the blue side of the spectra gets weaker with faster molecular dynamics. In the XXXX polarization condition, the intensity decrease is slightly more evident, while for the XXYY polarization condition the spectra simply get more symmetric with faster dynamics.
This observation indicates that the asymmetry of the 2DIR lineshapes is dominated by the excitonic mode structure, while the structural dynamics acts to average out these asymmetries.

\section{Concluding remarks}

Experimental and theoretical studies of the linear and twodimensional IR spectra of the amide I vibrational mode in neat liquid formamide are presented. The strong resonant vibrational interactions between neighboring amide I modes lead to delocalization and formation of largely extended vibrational excitons. The nonlinear vibrational signals are simulated with a recently developed method based on the numerical integration of the Schrödinger equation with the split operator technique for efficient calculation of the nonlinear response functions of fluctuating exciton systems. Both the linear and two-dimensional IR spectra are dominated by the intermolecular vibrational coupling, due to the insensitivity of the amide I mode to local structure and to the large interaction between the transition dipole moments. The excitonic character of the vibrational excitations results in the strong asymmetry observed in the linear and two-dimensional IR spectra. In the two-dimensional spectra and in their time evolution, a clear trend is visible, with the blue side of the spectrum showing larger antidiagonal broadening and faster loss of correlations. This effect is even more evident comparing different polarization conditions.

Two possible sources for the observed 2DIR lineshapes are discussed: different structural dynamics and cross-peak appearance featured by different coupling conditions of the excitonic modes on the red and blue sides of the spectrum. Even though both mechanisms contribute to the 2DIR signal, our analysis reveals the cross-peak formation as the major component contributing to the lineshape. Structural dynamics play a smaller role. Molecular dynamics simulation supports this interpretation, showing that the stronger coupling characterizing the high frequency part of the spectrum corresponds to excitonic states with larger size and significant overlap.

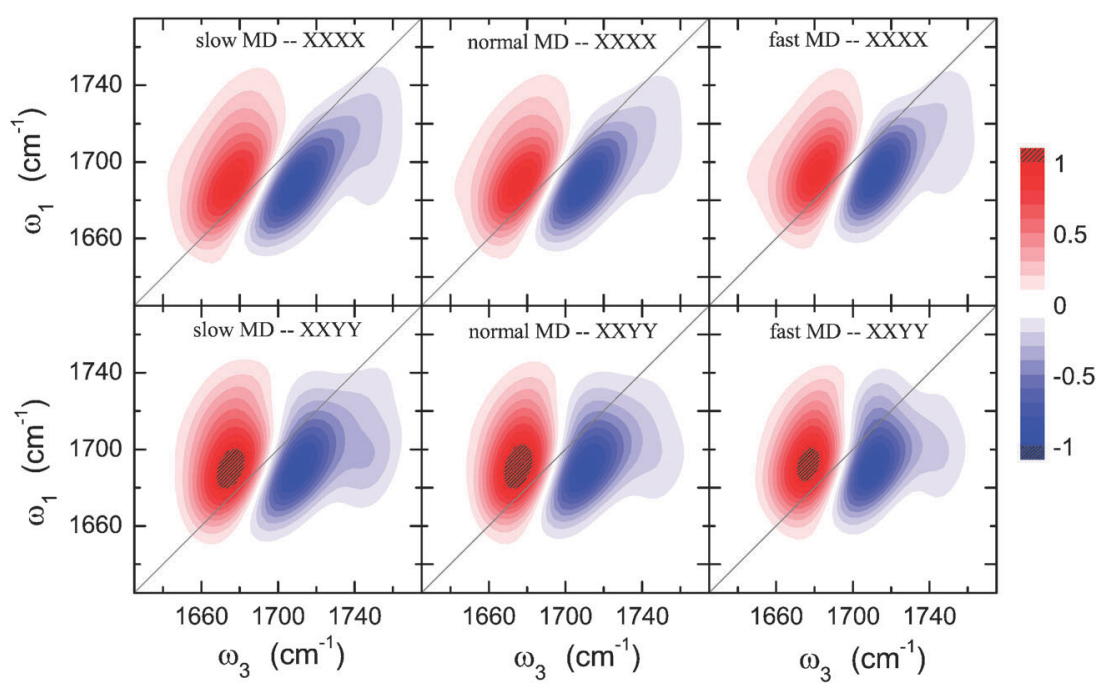

Fig. 8 2DIR spectra calculated for slow, normal and fast molecular dynamics in XXXX and XXYY polarization conditions (see panels). $\omega_{1}$ and $\omega_{3}$ refer to the excitation and detection frequencies, respectively. 


\section{Acknowledgements}

A. Paarmann gratefully acknowledges very helpful discussions with Hajime Torii (Department of Chemistry, Shizuoka University, Japan). The authors are grateful to Gianfranco Lauria (Department of Physics and LENS, University of Florence, Italy) for support in computer facilities at LENS. This work was supported by European Union Contract RII3-CT-2003506350. A. Paarmann was supported by the Natural Sciences and Engineering Research Council of Canada.

\section{References}

1 S. Mukamel, Annu. Rev. Phys. Chem., 2000, 51, 691.

2 D. M. Jonas, Annu. Rev. Phys. Chem., 2003, 54, 425.

3 M. Cho, Chem. Rev., 2008, 108, 1331.

4 P. Hamm, J. Helbing and J. Bredenbeck, Annu. Rev. Phys. Chem., 2008, 59, 291.

5 P. Hamm, M. Lim and R. M. Hochstrasser, J. Phys. Chem. B, 1998, 102, 6123.

6 M. F. DeCamp, L. DeFlores, J. M. McCracken, A. Tokmakoff, K. Kwac and M. Cho, J. Phys. Chem. B, 2005, 109, 11016.

7 I. V. Rubtsov, J. Wang and R. M. Hochstrasser, Proc. Natl. Acad. Sci. U. S. A., 2003, 100, 5601.

8 C. Kolano, J. Helbing, M. Kozinski, W. Sander and P. Hamm, Nature, 2006, 444, 469.

9 H. Maekawa and N.-H. Ge, J. Phys. Chem. B, 2010, 114, 1434.

10 A. G. Dijkstra, T. 1. C. Jansen and J. Knoester, J. Phys. Chem. A, 2010, 114, 7315.

11 T. 1. C. Jansen and J. Knoester, J. Phys. Chem. B, 2006, 110, 22910.

12 Z. Ganim and A. Tokmakoff, Biophys. J., 2006, 91, 2636.

13 T. 1. C. Jansen and J. Knoester, Biophys. J., 2008, 94, 1818.

14 J. Wang, W. Zhuang, S. Mukamel and R. Hochstrasser, J. Phys. Chem. B, 2008, 112, 5930 .

15 H. Torii, J. Phys. Chem. A, 2006, 110, 4822.

16 K. Kwac, H. Lee and M. Cho, J. Chem. Phys., 2004, 120, 1477.

17 V. V. Volkov and P. Hamm, Biophys. J., 2004, 87, 4213.

18 S. Sul, D. Karaiskaj, Y. Jiang and N.-H. Ge, J. Phys. Chem. B, 2006, 110, 19891.

19 N. Kobko and J. J. Dannenberg, J. Phys. Chem. A, 2003, 107, 6688.

20 Z. Ganim, H. S. Chung, A. W. Smith, L. P. DeFlores, K. C. Jones and A. Tokmakoff, Acc. Chem. Res., 2008, 41, 432.

21 H. S. Chung, Z. Ganim, K. C. Jones and A. Tokmakoff, Proc. Natl. Acad. Sci. U. S. A., 2007, 104, 14237.

22 L. P. DeFlores and A. Tokmakoff, J. Am. Chem. Soc., 2006, 128, 16520.

23 P. Mukherjee, I. Kass, I. T. Arkin and M. T. Zanni, Proc. Natl. Acad. Sci. U. S. A., 2006, 103, 3528.

24 A. G. Dijkstra and J. Knoester, J. Phys. Chem. B, 2005, 109, 9787.

25 J. Manor, P. Mukherjee, Y.-S. Lin, H. Leonov, J. L. Skinner, M. T. Zanni and I. T. Arkin, Structure, 2009, 17, 247.

26 A. T. Krummel, P. Mukherjee and M. T. Zanni, J. Phys. Chem. B, 2003, 107, 9165.

27 A. T. Krummel and M. T. Zanni, J. Phys. Chem. B, 2006, 110, 13991.

28 C. Lee, K.-H. Park, J.-A. Kim, S. Hahn and M. Cho, J. Chem. Phys., 2006, 125, 114510.

29 M. L. Cowan, B. D. Bruner, N. Huse, J. R. Dwyer, B. Chugh, E. T. J. Nibbering, T. Elsaesser and R. J. D. Miller, Nature, 2005, 434, 199.

30 D. Kraemer, M. L. Cowan, A. Paarmann, N. Huse, E. T. J. Nibbering, T. Elsaesser and R. J. D. Miller, Proc. Natl. Acad. Sci. U. S. A., 2008, $105,437$.

31 A. Paarmann, T. Hayashi, S. Mukamel and R. J. D. Miller, J. Chem. Phys., 2008, 128, 191103.

32 A. Paarmann, T. Hayashi, S. Mukamel and R. J. D. Miller, J. Chem. Phys., 2009, 130, 204110.

33 M. Lima, R. Chelli, V. V. Volkov and R. Righini, J. Chem. Phys., 2009, 130, 204518.
34 J. Stenger, D. Madsen, P. Hamm, E. T. J. Nibbering and T. Elsaesser, J. Phys. Chem. A, 2002, 106, 2341.

35 S. Yeremenko, M. S. Pshenichnikov and D. A. Wiersma, Chem. Phys. Lett., 2003, 369, 107.

36 C. J. Fecko, J. D. Eaves, J. J. Loparo, A. Tokmakoff and P. L. Geissler, Science, 2003, 301, 1698.

37 J. B. Asbury, T. Steinel, C. Stromberg, S. A. Corcelli, C. P. Lawrence, J. L. Skinner and M. D. Fayer, J. Phys. Chem. A, 2004, 108, 1107.

38 J.-H. Ha, Y. S. Kim and R. M. Hochstrasser, J. Chem. Phys., 2006, 124, 064508.

39 J. Park, J.-H. Ha and R. M. Hochstrasser, J. Chem. Phys., 2004, 121, 7281.

40 H. Torii, J. Phys. Chem. A, 2004, 108, 2103.

41 H. Torii and M. Tasumi, J. Phys. Chem. B, 1998, 102, 315.

42 A. Mortensen, O. Faurskov Nielsen, J. Yarwood and V. Shelley, Vib. Spectrosc., 1994, 8, 37.

43 M.-C. Bellissent-Funel, S. Nasr and L. Bosio, J. Chem. Phys., 1997, 106, 7913.

44 E. Tsuchida, J. Chem. Phys., 2004, 121, 4740.

45 Y. P. Puhovski, L. P. Safonova and B. M. Rode, J. Mol. Liq., 2003, 103-104, 15 .

46 Y. P. Puhovski and B. M. Rode, Chem. Phys., 1995, 190, 61.

47 Y. L. Li, L. Huang, R. J. D. Miller, T. Hasegawa and Y. Tanimura, J. Chem. Phys., 2008, 128, 234507.

48 J. Gao, J. J. Pavelites and D. Habibollazadeh, J. Phys. Chem., 1996, 100, 2689.

49 A. Mortensen, O. Faurskov Nielsen, J. Yarwood and V. Shelley, J. Phys. Chem., 1995, 99, 4435.

50 C. Perchard and J. P. Perchard, J. Raman Spectrosc., 1975, 3, 277.

51 W. Schindler, P. T. Sharko and J. Jonas, J. Chem. Phys., 1982, 76, 3493.

52 M. G. Giorgini, G. Fini and P. Mirone, J. Chem. Phys., 1983, 79, 639.

53 H. Torii and M. Tasumi, J. Chem. Phys., 1993, 99, 8459.

54 S. Mukamel, Principles of nonlinear optical spectroscopy, Oxford University Press, Oxford, 1995.

55 T. 1. C. Jansen, B. M. Auer, M. Yang and J. L. Skinner, J. Chem. Phys., 2010, 132, 224503.

56 T. Hayashi, T. l. C. Jansen, W. Zhuang and S. Mukamel, J. Phys. Chem. A, 2005, 109, 64.

57 T. 1. C. Jansen and J. Knoester, J. Chem. Phys., 2006, 124, 044502.

58 B. M. Auer and J. L. Skinner, J. Chem. Phys., 2007, 127, 104105.

59 B. M. Auer and J. L. Skinner, J. Chem. Phys., 2008, 128, 224511.

60 V. V. Volkov, R. Chelli, W. Zhuang, F. Nuti, Y. Takaoka, A. M. Papini, S. Mukamel and R. Righini, Proc. Natl. Acad. Sci. U. S. A., 2007, 104, 15323.

61 J. Stark and G. Wendt, Ann. Phys., 1914, 43, 983.

62 Note that the electric field acting on the $m$ th amide I mode is that generated by all charges but those belonging to the molecule $m$.

63 H. Torii and M. Tasumi, J. Chem. Phys., 1992, 96, 3379.

64 R. Chelli, V. V. Volkov and R. Righini, J. Comput. Chem., 2008, 29, 1507.

65 The ${ }^{12} \mathrm{C}$-labeled molecule in the simulated sample was chosen randomly.

66 S. Krimm and Y. Abe, Proc. Natl. Acad. Sci. U. S. A., 1972, 69, 2788.

67 W. H. Moore and S. Krimm, Proc. Natl. Acad. Sci. U. S. A., 1975, 72, 4933.

68 H. Torii, J. Phys. Chem. A, 2004, 108, 7272.

69 S. Marsili, G. F. Signorini, R. Chelli, M. Marchi and P. Procacci, J. Comput. Chem., 2010, 31, 1106.

70 R. Bloem, A. G. Dijkstra, T. 1. C. Jansen and J. Knoester, J. Chem. Phys., 2008, 129, 055101.

71 M. T. Zanni, N.-H. Ge, Y. S. Kim and R. M. Hochstrasser, Proc. Natl. Acad. Sci. U. S. A., 2001, 98, 11265.

72 The differences are taken after normalizing both XXXX and XXYY spectra to their respective positive maximum value.

73 This quantity was calculated from the MD simulation trajectory of 320 molecules published in ref. 33 .

74 Incidentally we note that $\omega_{\max }$ also corresponds to the frequency of maximum participation number. 